\title{
Resource deployment and donation allocation for epidemic outbreaks
}

\author{
Azrah Anparasan $^{1}$ (D) Miguel Lejeune ${ }^{2}$
}

Published online: 3 April 2017

(C) Springer Science+Business Media New York 2017

\begin{abstract}
Non-profit organizations play a central role in responding to the devastating consequences of epidemic outbreaks in developing economies. We propose an epidemic response model in resource-limited countries that determines the number, size, and location of treatment facilities, deploys critical medical staff, locates ambulances to triage points, and organizes the transportation of severely ill patients to treatment facilities. The model is based on the 2010 cholera outbreak in Haiti and is general enough to be used for similar epidemic outbreaks. The model enables not-for-profit decision-makers to assess health care triage capabilities, transportation needs, and requirements for medical personnel staffing and deployment. We propose an algorithmic procedure using hierarchical constraints and valid inequalities that reduce the solution time by one order of magnitude. Additionally, we propose a framework that can be used to optimally allocate a donation, to determine a list of priorities for earmarked donations, and to perform a cost-benefit analysis of an intervention strategy financed by a donation. The model is formulated as a large integer problem with many symmetries. An extended analysis based on the 2010 cholera outbreak in Haiti provides insights about: the criticality of the resources, the implementation of a balanced response strategy, the optimal allocation of resources in terms of the severity of the attack rate, and the benefits of the proposed response approach with respect to other intervention strategies.
\end{abstract}

Keywords Epidemic outbreak - Resource allocation for epidemics · Health care in developing countries $\cdot$ Medical facility location

$凶$ Azrah Anparasan

azrah@gwu.edu

Miguel Lejeune

mlejeune@gwu.edu

1 Department of Engineering Management and Systems Engineering, The George Washington University, Science and Engineering Hall, Suite B1800, 800, 22nd Street NW, Washington, DC 20052, USA

2 Decision Sciences Department, The George Washington University, Funger Hall, 406, 2201 G Street NW, Duquès Hall, Washington, DC 20052, USA 


\section{Introduction}

\subsection{Background and contributions}

The global burden of epidemics has increased tremendously over the past years. The 2014 Ebola outbreak in West Africa has thus far affected about 25,000 and claimed around 10,000 lives in Guinea, Liberia, and Sierra Leone (CDC 2015). The 2008 cholera outbreak in Zimbabwe peaked with over 8000 cases reported in a single week (WHO 2015a). Since the 2010 cholera outbreak in Haiti, over 600,000 cases were reported (by 2013) and 8000 lives were lost (Roos 2013).

In developing countries, consequences of epidemics are exacerbated due to poor water and sanitation conditions, lack of health care services, and economic challenges (UNICEF 2013; Lemonick 2011). Additionally, access to treatment facilities is impeded in remote locations due to high transportation costs and physical distance to treatment facilities (Farmer et al. 2011; McCoy and Johnson 2014). Long distances to treatment facilities contributed to approximately $40 \%$ of deaths at the initial onset of the cholera outbreak in Haiti (Farmer et al. 2011; Ivers et al. 2010).

The timely response to an epidemic outbreak is growing in importance, as climate change, population density, and urbanization can be catalysts that further aggravate the consequences of an epidemic. Control measures for epidemics can either be preventive (pre-event) and based on the design of an efficient response mechanism should a disease declare, or reactive (postevent) and aimed at controlling a confirmed outbreak (Dasaklis et al. 2012). In this paper, we propose an epidemic response model whose objective is to control an outbreak. More precisely, the model (1) determines the number, type, and location of treatment facilities, (2) staffs these facilities with medical personnel, (3) locates ambulances to triage points, and (4) organizes the transportation of patients to treatment centers. The model is based on the 2010 cholera outbreak in Haiti that happened shortly after the earthquake.

The model accounts for the scarcity of transportation resources and key medical personnel. These are critical issues in developing countries which are further compounded when the epidemic occurs in the aftermath of a natural disaster and further strains the limited resources. We adopt a "pull" modeling approach (AHRQ 2004) in the sense that people are transported to treatment centers to receive medication (instead of bringing the medicine directly to individuals). Cholera response standards and guidelines published by health care organizations such as the World Health Organization (WHO), the Centers for Disease Control and Prevention (CDC), and the ministry of health and population of Haiti (MSPP) are utilized to set the model.

The proposed model will support the epidemic-response operations conducted by governments, non-governmental organizations (NGOs), other non-profit agencies, and foundations. The proposed model will provide insights to non-profit organizations-often viewed as the main actors in the response to an epidemic outbreak (McLachlin et al. 2009)—about what is most needed (i.e., purchase of ambulances, medicine, recruiting of medical personnel, etc.) and on how to design their aid and donation policies, and in particular the selection of activities for which earmarked donations should be raised in priority.

This study addresses some gaps in the literature about epidemic control identified by Dasaklis et al. (2012). First, resource allocation models and contingency plans for epidemics often overlook key logistics parameters. Many models do not consider patient flow and the need to transport people to hospitals to receive medical treatment, which is particularly challenging for severe cases in rural areas (PAHO 2010). Our model accounts for the transportation requirements and the limited capacity of treatment centers as well as lim- 
ited availability of medicine. Second, Dasaklis et al. (2012) note that "research approaches addressing epidemics control logistics aspects in the aftermath of natural disasters are limited" despite the fact that epidemics involving diarrheal diseases often occur in the aftermath of a natural disaster. The increase in infectious disease outbreaks after natural disasters is due to prolonged after-effects of the disaster (e.g., displaced populations, overcrowded shelters, poor water and sanitation conditions, and limited access to healthcare services) (Kouadio et al. 2012). Effective site planning, provision for treatment and supportive care will help control the impact of outbreaks (Kouadio et al. 2012) following a natural disaster, and are addressed in this study. Third, the literature often assumes that the "the surge capacity of dispensing centers is infinite" (Dasaklis et al. 2012). The proposed model is cognizant of the limited number of beds in treatment centers and the limited availability of medicine and workforce. Fourth, the reliance upon standards and guidelines published by health care organizations reinforces the operationality of the model. Finally, this study also contributes to the literature on health care and humanitarian operations in resource-restricted economies (e.g., Kraiselburd and Yadav 2013; McCoy and Johnson 2014).

Besides the modeling contributions described above, we define a framework to optimally allocate a donation, to determine a list of priorities for earmarked donations, and to perform a cost-benefit analysis of an intervention strategy financed by a donation. We also develop an algorithmic procedure based on the introduction of hierarchical constraints that permits to solve efficiently this type of problems. The method is widely applicable to constrained resource allocation and deployment models with symmetries. Finally, an extended analysis based on the 2010 cholera outbreak in Haiti provides insights about: the criticality of the resources needed, the implementation of a balanced response strategy, the optimal allocation of resources in terms of the severity of the attack rate, the benefits of the intervention proposed by our model with other intervention strategies.

\subsection{Literature review}

The central theme of this study is the containment of an epidemic outbreak by not-forprofit organizations. We review below several streams of literature within this theme that are particularly relevant: siting of temporary medical facility locations, resource allocation and staffing during an epidemic outbreak, and health care and humanitarian operations in developing economies.

Locating accessible temporary facilities at the onset of an epidemic outbreak is paramount for effective and timely delivery of care. Two types of facilities - casualty collection points (CCPs) (Drezner 2004; Apte et al. 2015) and point of dispensing sites (PODs) (Lee et al. 2009) - are commonly relied upon. A CCP is stationary, located at a predetermined location, provides decontamination, emergency medical assessment, treatment, and, when necessary, onward transportation of victims of a mass casualty incident (Apte et al. 2015). PODs are also positioned in pre-determined locations but can be mobile. The location of PODs is chosen so that it can be easily accessed and it can service an entire region affected by a disease outbreak or facing the potential threat of a disease (Lee et al. 2009). CCPs have been used to plan for mass casualty events, such as a bomb blast or an earthquake (Drezner 2004). Apte et al. (2015) propose an optimization model that maximizes the number of casualties (i.e., the throughput) reaching CCPs. They identify the optimal CCP locations for a hypothetical bombing scenario in the District of Columbia, and determine the allocation of ambulances to transport casualties to hospitals, and staffing requirements for triage, administration, and other services. Lee et al. (2009) provide a decision support tool to determine POD sites for mass prophylaxis during an anthrax attack in a metropolitan area. The model incorporates coverage constraints to limit 
the travel distance, and provide bus services from designated pick up points to PODs. Lee et al. (2009) also calculate the public health personnel required to operate the PODs and the number of drivers needed. Compared to developed countries, developing ones must deal with additional complications and limitations in health care facility location problems (Rahman and Smith 2000). While the aforementioned studies concern disasters in major US cities, the proposed model takes into account epidemic control challenges arising in the immediate aftermath of a natural disaster in a resource-restricted country, and is designed for the entire country. In particular, the model considers the limited availability of doctors and nurses and is in line with the staggering scarcity of medical personnel in developing economies in general (World Bank 2015).

The importance of utilizing operations research methods to support decisions in disaster operations management has been strongly emphasized (see, e.g., Wassenhove and Pedraza Martinez 2012; Altay and Green 2006). Regarding epidemics, several models focused on the allocation and distribution of medical resources have been proposed (Koyuncu and Erol 2010; Rachaniotis et al. 2012; Aaby et al. 2006). Sobieraj et al. (2007) allocate physical resources, such as beds and ventilators, during mild and severe influenza outbreaks on the basis of existing emergency response plans. Koyuncu and Erol (2010) provide beds and ventilators under limited budget for the case of pandemic influenza in Turkey. Aaby et al. (2006) develop a simulation model to plan mass dispensing and vaccination clinic operations in the event of a sudden outbreak of a pandemic in a Maryland county. There is no specified limit on the number of personnel available which is allocated on the basis of specific functions (e.g., registration, consultation, vaccination). Lee et al. (2009) determine the need for public health workers in order to man POD facilities to be opened in the event of an influenza outbreak in Atlanta. They assume that public health personnel are volunteers and have no restriction in supply. To our knowledge, there is no model which considers jointly the allocation of limited medical (staff and medicine) and transportation resources, and the transportation of patients to treatment centers, which is one of the features of the proposed model.

A number of studies about health care and humanitarian operations in resource-limited countries have recently appeared. McCoy and Johnson (2014) propose a clinic capacity optimization model that shows the importance of accounting for adherence into clinic planning decisions and test it on HIV epidemic case study in Zambia. Kraiselburd and Yadav (2013) highlight how ineffective supply chains are a barrier to health care delivery in resourcerestricted areas and emphasize the need for future research in this area. McCoy and Lee (2014) study how inefficiency and inequity hamper humanitarian operations and health delivery in resource-restricted economies. They show how the non-profit organization Riders for Health helps provide reliable transportation to health workers in rural parts of sub-Saharan Africa.

The paper is organized as follows. The next section presents the model and justifies its assumptions. Section 3 describes the algorithmic framework used to efficiently solve the model. Section 4 describes the data sources. Section 5 analyzes the results and discusses their meaning and possible use by non-profit organizations. Section 6 evaluates the scalability of the algorithmic method, while Sect. 7 provides concluding remarks.

\section{Problem description}

In this section, we first describe the characteristics of a cholera epidemic outbreak before presenting the model that is motivated by the cholera outbreak in Haiti, a country with very poorly coordinated disaster response operations (Altay and Labonte 2014). 
Cholera is an acute diarrheal disease that is treatable with oral rehydration solutions (ORS) provided at triage points. However, severe cholera cases require treatment using antibiotics and intravenous solutions (IVs) provided in cholera treatment facilities (WHO 2015a; MSPP and CDC 2011). Unlike diseases such as influenza, cholera is not transmitted from direct person-to-person contact (non-contagious). The transmission of the virus occurs through infected food and water. If a patient remains untreated, he/she has more chances of contaminating the surrounding area due to fecal contamination under poor sanitation conditions. Differing from tuberculosis and other infectious diseases (McCoy and Johnson 2014), cholera treatment does not require the adherence to a prolonged regimen. Next, we motivate and formulate an epidemic response model for a cholera outbreak scenario in a resource-limited country.

\subsection{Modeling assumptions}

\subsubsection{Standards and guidelines of health care organizations}

According to the MSPP and CDC, three types of treatment facilities are established during a cholera outbreak: Cholera Treatment Centers (CTCs), Cholera Treatment Units (CTUs) and Oral Rehydration Points (ORPs) (MSPP and CDC 2011). CTCs are the largest in terms of treatment capacity and are set up as part of an existing facility or in a temporary tent set up in a suitable location (Fisher et al. 2014; WHO 2004). A CTC can have from 50 to 200 beds, while a CTU will have a capacity of 20 to 30 beds (MSPP and CDC 2011). Humanitarian practice considers that such treatment facilities can be set up within very short periods of time (WHO 2004). The decision to open a facility depends on the availability of medical staff and the staffing requirements (see MSPP and CDC 2011; UN 2010) for CTCs and CTUs to be operational. An ORP is a satellite station where patients are triaged (triage points) to assess their condition. Milder forms of dehydration can be treated at such a location. The model locates ambulances at the triage points. If an incoming patient is severely dehydrated, she/he is transported to a CTU or CTC from the triage locations. Since ORPs are easy to setup and operate, we assume that they are uncapacitated (see, e.g., MSPP and CDC (2011); Farmer et al. (2011)).

Physicians and nurses are the two indispensable types of medical personnel to be assigned to treatment facilities (MSPP and CDC 2011). MSPP and CDC (2011) and UN (2010) provide a detailed breakdown of nurse and physician staffing requirements for different capacities of CTCs and CTUs (see Sect. 4.3). MSPP provides the number of cases in the ten Haitian departments (MSPP 2011) from which we estimate the number of severely ill patients (Sect. 4.1).

\subsubsection{Context-based assumptions}

Haiti has ten administrational departments and these departments are further broken down to 42 arrondissements. We consider one central triage location (ORP) for each arrondissement.

Once responding organizations that set up temporary treatment facilities complete their missions, there is a serious risk that the public health system returns to its pre-epidemic state, thus impeding the continuity of operations (Farmer et al. 2011). Our model locates treatment facilities in or near hospitals, health centers, and field hospital locations to avoid this issue. We consider 382 prospective treatment facility location points across the country (see Sect. 4.2 for details). 
The number of available ambulances was very limited at the time of the cholera outbreak in Haiti (Farmer et al. 2011; Ivers et al. 2010). In 2014, only about 50 ambulances were available in Haiti (PRI 2014). Therefore, we assume fewer (i.e., 35) available ambulances in this study.

Cholera, with an incubation period of only $2 \mathrm{~h}$ to 5 days (Lemonick 2011), is capable of killing patients within hours in extreme cases (WHO 2015a). Therefore, facilities must be located within a limited driving distance from triage locations. With the same objective of reducing the time for a patient to reach a treatment center, we assign the ambulances to triage locations and not to CTCs and CTUs.

\subsection{Model formulation}

We propose an integer linear programming (ILP) epidemic response model for a cholera outbreak in the aftermath of a natural disaster. The model (1) determines the number, size, type (CTCs and CTUs), and location of cholera treatment facilities, (2) deploys critical medical care personnel (physicians and nurses) across the opened facilities, (3) locates ambulances at triage locations, and (4) organizes the transportation of the geographically-dispersed severely ill patients to treatment facilities. Haiti lacked transportation resources and qualified medical personnel. The model captures these issues faced by a resource-restricted country hit by an epidemic. While based on the 2010 cholera outbreak in Haiti, the model is generalizable to other cases of epidemic outbreaks in resource-restricted economies. Table 1 summarizes the notations used in the model.

\subsubsection{Objective function}

The usual epidemic containment goals are the reduction in the rate of susceptibles contracting the disease and in the mortality rate of those already infected (Dasaklis et al. 2012). In this model, we maximize the number of severely ill patients transported from triage locations to medical facilities where they can receive appropriate treatment:

$$
\max z=\sum_{i \in I} \sum_{j \in J_{i}} \sum_{k \in K} s_{i j k}
$$

We do not consider the transportation of mild cholera cases, since they can be successfully treated with rehydration packages at triage locations. The objective is a proxy for the minimization of the fatality rate, i.e., number of overall deaths due to cholera.

\subsubsection{Constraints}

The number of severely ill patients transported from a triage location to any treatment facility cannot exceed the total number $p_{i}$ of severely ill patients at a triage location $i$ :

$$
\sum_{j \in J_{i}} \sum_{k \in K} s_{i j k} \leq p_{i} \quad \forall i \in I
$$

Constraint (3) specifies that the number of severely ill patients treated in a facility location $j$ is limited by the medical personnel $\sum_{r \in R} z_{j r}$ available at this location. Some non-severely ill patients can also arrive at treatment facilities walking or using personal transport and severely ill patients may have to stay more than 1 day. Additionally, we consider the case of an epidemic in the aftermath of a natural disaster, which further strains the availability of 
Table 1 Notation

\begin{tabular}{|c|c|}
\hline \multicolumn{2}{|l|}{ Sets } \\
\hline$I$ & Set of triage locations \\
\hline$J$ & Set of treatment locations \\
\hline$J_{i}$ & Set of treatment locations within specified travel time from triage location $i$ \\
\hline$I_{j}$ & Set of triage locations within specified travel time from facility location $j$ \\
\hline$L$ & Set of treatment facility types $\{C T C, C T U\}$ \\
\hline$K$ & Set of ambulances \\
\hline$R$ & Set of medical staff types $\{$ doctors, nurses $\}$ \\
\hline$D$ & Set of departments in Haiti $\{1 \ldots 10\}$ \\
\hline$B$ & Allocated budget amount \\
\hline \multicolumn{2}{|c|}{ Parameters } \\
\hline$p_{i}$ & Average number of severely ill people at triage location $i$ \\
\hline$t_{i j}$ & Time (in hour) to drive from triage location $i$ to treatment facility location $j$ \\
\hline$c l$ & Maximum capacity of treatment facility of type $l$ \\
\hline$B$ & Seating capacity per ambulance \\
\hline$Q$ & Initial number of ambulances available \\
\hline$u_{r}$ & Number of patients that one medical staff person of type $r$ can treat daily \\
\hline$m_{r}$ & Initial availability of medical staff of type $r$ \\
\hline$n \_\max _{l r}$ & Number of medical staff type $r$ required for facility type $l$ to be fully operational \\
\hline$n \_\min _{l r}$ & Minimum number of medical staff of type $r$ for facility type $l$ to be operational \\
\hline$e_{l}$ & Stock of medicine available (units) per treatment facility type $l$ \\
\hline$\alpha$ & Percentage of time that medical staff spend on severely ill patients per day \\
\hline$\beta$ & Fraction of bed capacity for severely ill patients in a treatment facility \\
\hline$\gamma_{i j}$ & Transfer time from $i$ to $j$ and time spent in traffic (in hours) \\
\hline \multicolumn{2}{|c|}{ Decision variables } \\
\hline$s_{i j k}$ & $\begin{array}{l}\text { Number of severely ill patients transported with ambulance } k \text { from triage location } i \\
\text { to facility location } j \text { that is within admissible distance }\end{array}$ \\
\hline$x_{j l}$ & Binary variable, 1 if facility type $l$ is located at location $j$, and 0 otherwise \\
\hline$y_{i k}$ & Binary variable, 1 if ambulance $k$ is located at triage location $i$, and 0 otherwise \\
\hline$w_{i j k}$ & Number of trips by ambulance $k$ from triage location $i$ to treatment facility $j$ \\
\hline$z_{j r}$ & Number of medical personnel of type $r$ assigned to facility location $j$ \\
\hline
\end{tabular}

physicians and nurses. Therefore, we assume that the medical staff can only devote a certain fraction $\alpha$ of their time to severely ill patients. The product $z_{j r} u_{r} \alpha$ denotes the maximum number of severely ill patients that can be treated in facility $j$ :

$$
\sum_{i \in I_{j}} \sum_{k \in K} s_{i j k} \leq \sum_{r \in R} z_{j r} * u_{r} * \alpha \quad \forall j \in J
$$

Similarly, it cannot be expected that the entire capacity of a treatment facility is constantly available to receive incoming severely ill patients. We denote by $(1-\beta)$ the busy proportion of a treatment facility, and consider in constraint (4) that only a fraction $\beta$ of the beds in a 
CTC or CTU are available for new severely ill cases:

$$
\sum_{i \in I_{j}} \sum_{k \in K} s_{i j k} \leq \sum_{l \in L} x_{i l} * c_{l} * \beta \quad \forall j \in J
$$

Constraint (5) specifies that the number of patients traveling in an ambulance from a triage location to a treatment facility cannot exceed the capacity of an ambulance:

$$
s_{i j k} \leq b * w_{i j k} \quad \forall i \in I, j \in J_{i}, k \in K
$$

Constraint (6) ensures that there can be at most one treatment facility opened at each candidate location. Constraint (7) makes sure that the number of patients that can be treated at a treatment facility $j$ is limited by the available stock of medicine.

$$
\begin{aligned}
& \sum_{l \in L} x_{j l} \leq 1 \quad \forall j \in J \\
& \sum_{i \in I_{j}} \sum_{k \in K} s_{i j k} \leq \sum_{l \in L} x_{j l} * e_{l} \quad \forall j \in J
\end{aligned}
$$

The total number of trips that an ambulance can make to any facility during a single period is limited by the number of hours of operation (i.e., $12 \mathrm{~h}$ ). This requirement is enforced by constraint (8), in which $2 t_{i j}$ denotes the time for the round trip between $i$ and $j$ and $\gamma_{i j}$ is the sum of the transfer time and the time lost due to traffic. The transfer time is a function of the distance between locations and the speed of the vehicle. Constraint (9) ensures that the number of ambulances allocated to triage locations does not exceed the number $q$ of available ambulances. Constraint (10) ensures that each ambulance is assigned to no more than one triage location.

$$
\begin{aligned}
& \sum_{j \in J_{i}} w_{i j k} *\left(2 t_{i j}+\gamma_{i j}\right) \leq 12 y_{i k} \quad \forall i \in I, k \in K \\
& \sum_{i \in I} \sum_{k \in K} y_{i k} \leq q \\
& \sum_{i \in I} y_{i k} \leq 1 \quad \forall k \in K
\end{aligned}
$$

The number of medical personnel needed by each type of treatment facility to function at its maximal capacity is specified by MSPP and CDC (2011). This is modelled with constraint (11) in which the number of medical personnel of each type $r$ allocated to a facility of type $l$ is restricted by $n \_\max _{l r}$. Additionally, for a facility to be operational, a minimum staffing requirement defined by $n \_$min $_{l r}$ enforced by (12) must be achieved:

$$
\begin{array}{ll}
z_{j r} \leq \sum_{l \in L} x_{j i} * n_{-} \max _{l r} & \forall j \in J, r \in R \\
z_{j r} \leq \sum_{l \in L} x_{j i} * n_{-} \text {min }_{l r} & \forall j \in J, r \in R
\end{array}
$$

Constraint (13) enforces the limited availability of medical personnel. The integrality restrictions are given by (14).

$$
\begin{aligned}
& \sum_{j \in J} z_{j r} \leq m_{r} \quad \forall r \in R \\
& s_{i j k}, w_{i j k}, z_{j r} \in \mathbb{Z}^{+} \quad \forall i \in I, j \in J_{i}, k \in K, r \in R
\end{aligned}
$$


We shall thereafter refer to the model minimizing (1) subject to the constraints (2)-(14) as M1.

\section{Algorithmic method}

The optimization model presented in Sect. 3 is for the entire country and is a complex integer linear programming problem which includes a large number of binary and general integer decision variables. Its size and structure pose serious computational challenges. It is thus crucial to design formulations that are not only mathematically valid but also computationally tractable.

The first challenge with the proposed formulation is to obtain the optimal solution in a reasonable amount of time. The second one, as confirmed by preliminary tests, is that the size of the branch-and-bound tree used for its solution can become so large for some problem instances that it exceeds a computer's memory capacity. In order to alleviate those issues, we derive in the next sub-sections hierarchical constraints that reduce the symmetry in the formulation and valid inequalities that strengthen the formulation. We evaluate in Sect. 6 the computational benefits of the valid inequalities and symmetry-breaking constraints.

\subsection{Hierarchical constraints}

Integer optimization problems with symmetries have particular structures that produce an exponential number of equivalent, symmetric solutions by means of re-indexation (Ghoniem and Sherali 2011). Optimization solvers become typically entangled in the wasteful exploration of equivalent solutions and it is thus important to detect, and possibly break, the symmetries in the formulation. Integer problems are typically solved with branch-and-bound or branch-and-cut algorithms that converge slowly in presence of symmetries, since many leaf nodes in the branch-and-bound tree can include symmetric global optima. Hierarchical constraints (Sherali and Smith 2001), isomorphism techniques (Margot 2010), and objective perturbations (Ghoniem and Sherali 2011) have been proposed to break symmetry.

In this problem, we have symmetry among ambulances for which we derive hierarchical symmetry-defeating constraints. We have to allocate a number $q$ of identical ambulances to $|I|$ triage locations. The ambulance symmetry-defeating constraints read

$$
\sum_{i^{\prime} \in I: i^{\prime} \leq i} y_{i^{\prime} k} \geq \sum_{i^{\prime} \in I: i^{\prime} \leq i} y_{i^{\prime} k+1} \quad \forall i \in I, k=1, \ldots q-1
$$

and stipulate that if an ambulance is assigned to triage location $i$, this ambulance must be the currently unassigned ambulance with the smallest index $k$. For example, if triage location with index $i=1$ is to be assigned an ambulance, the above constraint forces this ambulance to be the one with index $k=1$. Such constraints are called hierarchical in the sense that they give the symmetric elements more distinct identities with the ranking defined by (15). The above constraints cut at least $|I| *(q-1) *(q-2) / 2$ symmetric solutions, and have thereby great potential to improve algorithmic performance. The incorporation of the hierarchical constraints in the formulation reduces the feasible region but preserves at least one optimal solution to the original problem, thereby (i) potentially reducing the search effort and (ii) guaranteeing that an optimal solution can be found using an exact solution approach. Observe finally that any permutation of the terms in the hierarchical constraint set provides an alternative admissible ranking of the symmetric elements. 


\subsection{Family of valid inequalities}

Valid inequalities permit to obtain stronger formulations. The set of fractional feasible solutions for the continuous relaxation including the valid inequalities is a strict subset of the same set for the continuous relaxation of the original formulation. Note that symmetry breaking constraints are not in general valid cuts, since they can off some symmetric equivalent integral points. However, they guarantee feasibility of at least one integral point per equivalence class and ensure that the optimal value can be reached.

In this paper, we derive four families of valid inequalities. The first one associates the variables $y_{i k}$ defining the assignment of ambulances to triage locations and the number $w_{i j k}$ of trips per ambulance and per pair of locations:

$$
y_{i k}\left\lceil\frac{p_{i}}{b}\right\rceil \leq \sum_{j \in J i} w_{i j k} \leq y_{i k} p_{i} \quad \forall i \in I, k \in K
$$

These valid inequalities fix the number of trips originating from triage location $i$ with ambulance $k$ to zero if ambulance $k$ is not assigned to $i$. Additionally, if ambulance $k$ is assigned to $i$, the above constraints define a tight lower bound on the number of trips with ambulance $k$ from $i$ to the health centers located within acceptable distance. The lower bound is defined as the ceiling of the ratio of the number $p_{i}$ of severely ill patients at $i$ to the capacity $b$ of an ambulance.

The second family of valid inequalities defines for each ambulance an upper bound on the number of trips between each pair of locations in terms of the number of patients transported and the capacity of an ambulance:

$$
w_{i j k} \leq \frac{s_{i j k}}{b}+1 \quad \forall i \in I, j \in J_{i}, k \in K
$$

The third family of valid inequalities requires each binary variable $y_{i k}$ defining the assignment of ambulances to triage locations to be 0 if no patient is transported from location $i$ :

$$
y_{i k} \leq \sum_{j \in J_{i}} s_{i j k} \quad \forall i \in I, k \in K
$$

The fourth set of inequalities stipulates that no patient can be transported to a potential treatment center site where no nurse or physician is deployed

$$
w_{i j k} \leq N z_{j r} \quad \forall i \in I, j \in J_{i}, k \in K, r \in R
$$

with $N$ a sufficiently large positive number.

\section{Data}

The data used in this research have been collected from various sources, such as humanitarian organizations, journal publications and health care organizations (WHO, CDC, and MSPP).

\subsection{Number of severely ill patients}

MSPP (2011) provides daily and cumulative data relevant to patient numbers and number of deaths per department from the beginning of the outbreak (i.e., late October 2010) until now. The patient data are provided for the ten departments in Haiti. We allocate the reported cases to each arrondissement proportionally to its population (Geohive 2015). Next, we assess the 
Table 2 Staffing requirements for cholera treatment facilities

\begin{tabular}{lll}
\hline Facility type: capacity & Minimum operational requirement & Full operational capacity \\
\hline CTC: 100 beds & Physician: 1 ; nurse: 5 & Physician: 6 ; nurse: 30 \\
CTU: 20 beds & Physician: $1 ;$ nurse: 1 & Physician: $2 ;$ nurse: 6 \\
\hline
\end{tabular}

number of severely ill patients based on the reported cases. In the base case of this study, we adopt WHO's view according to which $20 \%$ of the cases are severe ones. Other estimates of the proportion of severely ill patients are analyzed in Sect. 6. Using the numbers of patients reported by MSPP allows us to develop a descriptive model to identify the critical resources required for an epidemic outbreak of similar nature.

\subsection{Candidate medical facility locations}

Cholera treatment facilities (i.e., CTCs and CTUs) can be properly set up in existing health facilities (MSPP and CDC 2011). Field hospitals, health centers with beds, and hospitals are prospective locations to set up treatment facilities. This amounts to a total of 382 candidates, whose locations are made available by the Pan American Health Organization (PAHO 2010). Each arrondissement has one aggregated central triage location (ORP). We have computed the actual driving distance between each pair of triage and treatment facility locations using the Google Distance Matrix API (Google 2015).

\subsection{Medical personnel staffing for CTC and CTU treatment facilities}

In accordance with medical standards, we assume that each CTC has a 100-bed capacity and that each CTU has a 20-bed capacity. Table 2 provides the number of medical personnel needed for a treatment facility to operate at full capacity as specified by MSPP and CDC (2011).

\subsection{Availability of medical personnel}

Haiti has very limited resources in terms of medical personnel (Farmer et al. 2011; Ivers et al. 2010). There are many disparate sources (e.g., NGOs) that report the number of medical personnel allocated to the cholera response in Haiti (Lopez and Valido 2011; MSF 2011). UNDP reports that in 2009, Haiti had a local medical staff capacity of 1 doctor and 1.8 nurses per 1000 population (UNDP 2009). Based on the Haitian population in 2009, we conservatively assume that $5 \%$ of the local pool is readily available for staffing treatment facilities at the initial outbreak phase (i.e., 45 doctors and 81 nurses). We consider other staffing levels in the analysis section.

\subsection{Ambulance speed and capacity}

The speed of the ambulances deployed in developing countries is affected by the road and traffic conditions. Harewood (2002) considers a range from 10 to $80 \mathrm{~km} / \mathrm{h}$ for emergency ambulance in Barbados. In this paper, we assume a speed of $25 \mathrm{~km} / \mathrm{h}$, accounting for the possible deterioration of the transportation conditions due to the natural disaster. We assume here that ambulances can transport up to five patients at the same time. 
Table 3 Values of parameters in the base case scenario

\begin{tabular}{llll}
\hline$\sum_{i \in I} p_{i}$ & 443 & $n \_\max _{l r}$ & $\{630 ; 26\}$ \\
$c_{l}$ & $\{20,100\}$ & $n \_\min _{l r}$ & $\{15 ; 11\}$ \\
$b$ & 5 & $e_{l}$ & $\{20,100\}$ \\
$q$ & 35 & $\alpha$ & $80 \%$ \\
$u_{r}$ & $\{2,3\}$ & $\beta$ & $80 \%$ \\
$m_{r}$ & $\{45,81\}$ & $\gamma_{i j}$ & $0.2 t_{i j}+0.25$ \\
\hline
\end{tabular}

\section{Analysis}

We present in Sect. 5.1 a base case scenario used as a benchmark to evaluate our model. In Sect. 5.2, we evaluate the criticality of the resources needed to respond to an epidemic. In Sect. 5.3, we provide guidelines for and assess the consequences on the overall welfare of delivering a balanced response across the country. Section 5.4 defines the optimal allocation of resources in terms of the severity of the attack rate. Section 5.5 proposes a framework for optimally allocating a donation and to determine a list of priorities for earmarked donations. Section 5.6 compares the benefits of the intervention proposed in this paper with other intervention strategies. Section 5.7 presents a cost-benefit analysis and estimates the value of a donation.

\subsection{Base case scenario}

We first define and illustrate the results for the base case scenario for Haiti's cholera outbreak. Table 3 gives a summary of the base case parameter settings.

The daily number of severely ill patients across the country is equal to 443 and represents $20 \%$ of the daily average number of cholera cases over the period ranging from 20th October 2010 to 31st Dec. 2010 (MSPP 2011). We set the maximum travel time to $1 \mathrm{~h}$. The speed of an ambulance is set to $25 \mathrm{~km} / \mathrm{h}$. Ambulances take $\left(2 t_{i j}+\gamma_{i j}\right)$ to make a round-trip from a triage location to a treatment facility, where $\gamma_{i j}$ includes a 15-min patient transfer time and additional transportation time due to traffic and deteriorated road conditions, which is set to $20 \%$ of the travel time $t_{i j}$ between $i$ and $j$. We set the fraction of time $\alpha$ that medical staff devotes to severely ill patients, and the fraction of beds $\beta$ allocated to severely ill patients to $80 \%$.

The model includes 43,844 integer variables (7554 are binary and 36,300 general integer) and 26,121 constraints and can be solved to optimality in less than $5 \mathrm{~min}$ with the proposed method. The results obtained by solving the integer programming problem M1 for the base case scenario are discussed below and summarized in Table 4. The computational aspects are discussed in Sect. 6.

A total of 266 severely ill patients (about $60 \%$ ) are transported to treatment facilities. All available physicians and nurses are deployed at the 4 CTCs and 15 CTUs opened. None of the CTCs and CTUs is staffed to operate at maximal capacity. Consequently, the average throughput, defined as the number of severely ill patients treated in a facility, is not maximal and amounts to 45 and 6 patients for CTCs and CTUs respectively. Only 23 out of the 35 available ambulances are used. Using more of them does not increase the number of treated patients, as the performance of the health care system is severely limited in our setting by the few nurses and physicians available. The average transportation time for a patient is approximately $33 \mathrm{~min}$. 
Table 4 Results for base case with model M1

Total number of severely ill patients treated 266

Number of medical staff utilized \{physicians, nurses

Average number of medical staff per CTC \{physicians, nurses

Average number of medical staff per CTU \{physicians, nurses

Number of treatment facilities opened \{CTCs, CTUs

Average throughput per treatment facility type $\{$ CTCs, CTUs $\}$

Average transportation time per patient in hours

$33 \mathrm{~min}$

Number of utilized ambulances

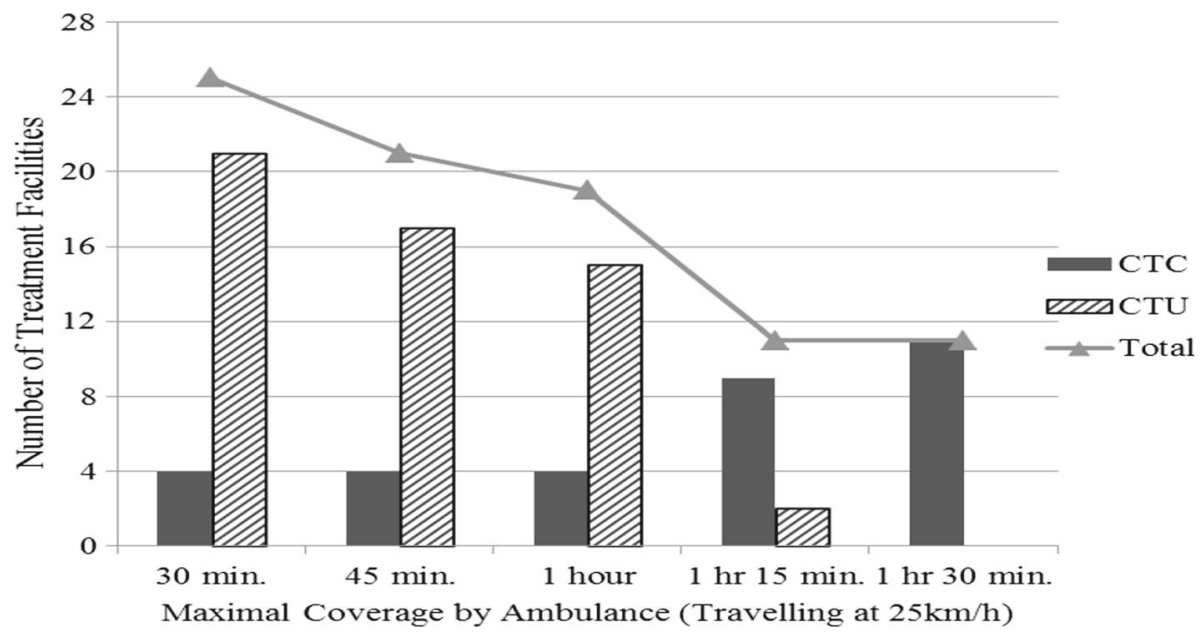

Fig. 1 Impact of coverage requirement on number of treatment facilities

\subsection{Criticality of resources}

In this section, we study the impact of the limited and binding availability of the resources on the number of sick people that can be treated. We first analyze the impact of the coverage requirement (i.e., travel time between triage location and treatment facility) on the number and types of opened facilities and the average travel time for patients. We consider various coverage levels ranging from 30 to $90 \mathrm{~min}$ with 15 -min increments. All other parameters are maintained at the base case levels. Figure 1 shows the variation in the number and type of treatment facilities.

Note that the maximum number of severely ill patients that can be treated is 266 under all coverage levels. This has two implications. First, since a reduced and hence more demanding coverage distance does not reduce the number of patients treated, it is thus preferable to opt for the shortest coverage distance. This reduces the overall time before a patient receives treatment, which is critical due to the short incubation period. In light of Fig. 2, locating more CTUs within a 30-min coverage distance provides the quickest access to treatment for severely ill patients. Figure 2 shows how the average patient transportation time varies with the coverage requirement. The extended times for the coverage requirement results in larger average transportation times. The second implication is that the optimal solutions of the 


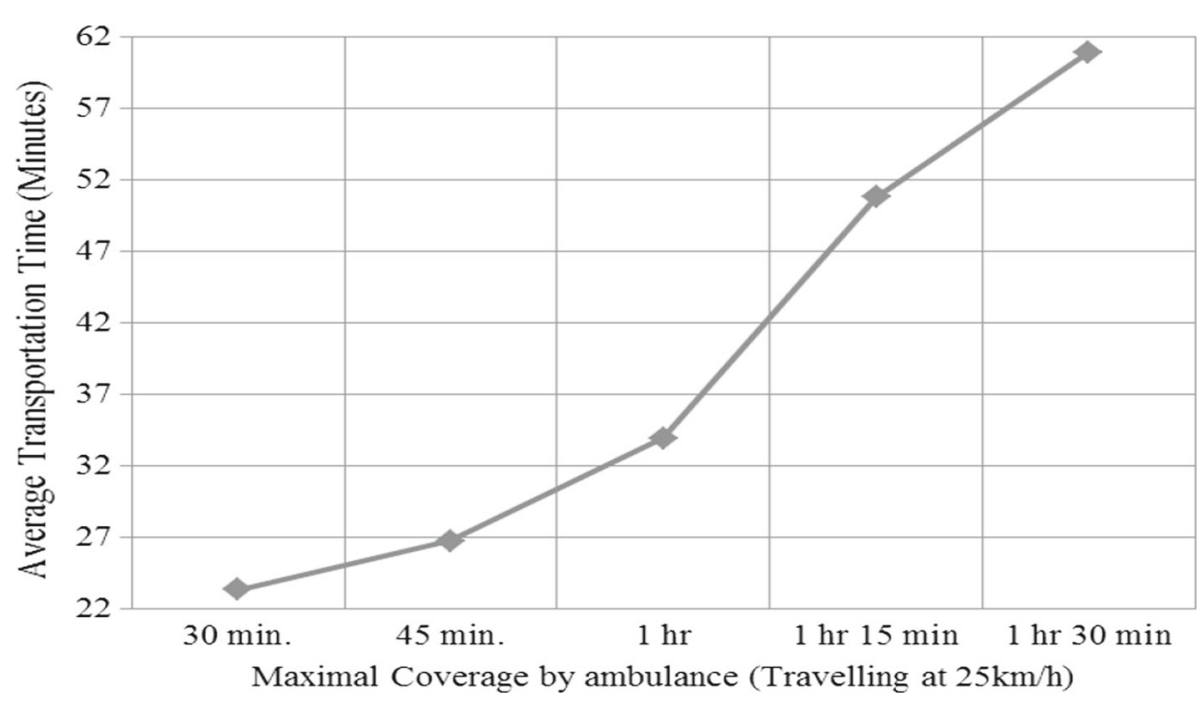

Fig. 2 Average transportation time as a function of coverage

problems for all the considered coverage limits are feasible and optimal for the problem with the 90-min coverage requirement. However, the converse is not true. The optimal solution reported in Fig. 1 for the problem with the 90-min coverage condition is not feasible for any of the more restrictive (i.e., 30, 45, $60 \mathrm{~min}$ ) coverage cases (the same applies to 60-min vs 30 and $45 \mathrm{~min}$ and to $45 \mathrm{vs} 30 \mathrm{~min}$ ). It follows therefore (see Fig. 1) that the total number of facilities is in general a decreasing function of the coverage time. The number of CTUs in particular increases as the time coverage condition decreases. This is in line with Lee et al.'s finding (2009) that the number of opened PODs diminishes when the travel distance increases.

Interestingly, it appears that between $7(20 \%)$ and $12(34 \%)$ of the 35 available ambulances are not used. This is somewhat an unexpected result as some of the literature about the Haiti cholera epidemic attributes a large number of deaths to the lack of transportation means (Farmer et al. 2011; Ivers et al. 2010). While our results do not negate the lack of medical transportation vehicles, they indicate that, under the considered scenario, ambulance availability is not the most binding resource and that the most critical resources are the limited availability of nurses and physicians.

\subsection{Balanced treatment policy}

Model M1 does not include any restriction about the allocation of the ambulances. This can lead some very unbalanced and somewhat unfair solutions. For example, some optimal solutions of M1 for the base scenario are such that no treatment facilities are opened in some Haitian departments and that none of their patients are treated. In order to address this issue, we add a service level constraint in model M1 that requires a certain percentage $\delta$ of the severely ill patients must receive treatment in each department (i.e., except those that have very few daily severely ill patients):

$$
\sum_{i \in I} p_{i} * \theta_{i d} * \delta \leq \sum_{i \in I} \sum_{j \in J_{i}} \sum_{k \in K} s_{i j k} * \theta_{i d} \quad \forall d \in D
$$




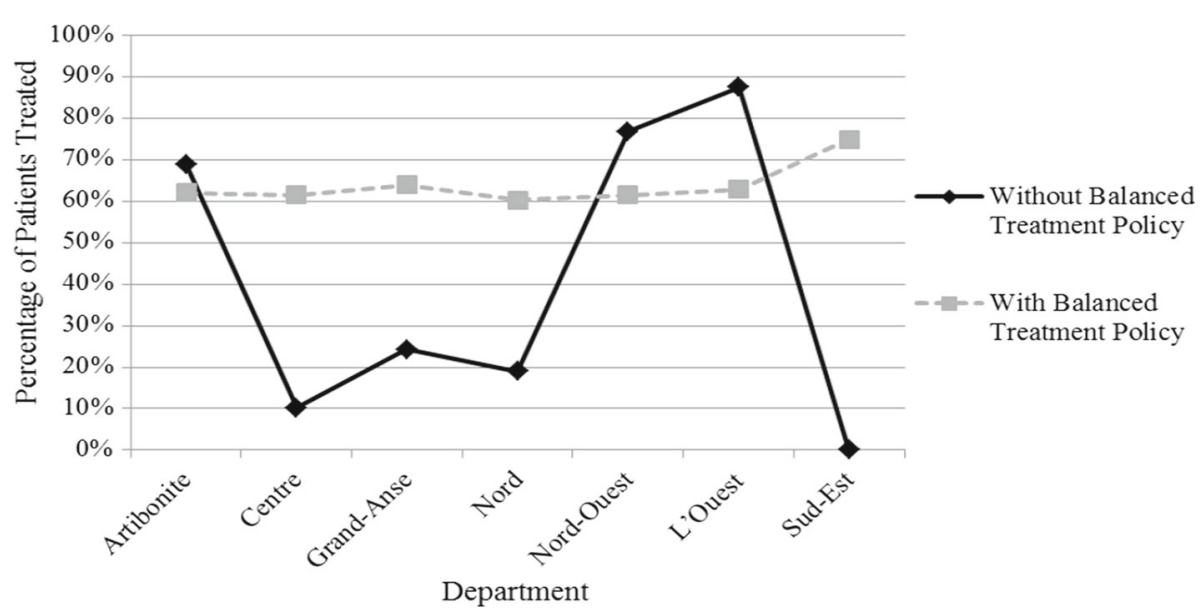

Fig. 3 Percentage of patients treated with and without balanced treatment policy

where $\theta_{i d}$ is a binary parameter equal to 1 if $i$ is in department $d$, and to 0 otherwise. We refer to the problem maximizing (1) under the constraints (1)-(14); (20) as model M1B.

Figure 3 displays for each department the percentages of severely ill patients treated with models M1 and M1B with $\delta=60 \%$. As expected, the proportions of patients treated in each department fluctuate strongly in the solutions of the two models; the solution given by M1B is much more balanced and fairer response across departments.

Most interestingly, the total number of patients is the same with both M1 and M1B, which means that the type of balance or equitable treatment policy enforced through (20) does not affect the overall welfare and does not result in a reduction of the number of patients treated across the country. However, enforcing a value of $\delta>61 \%$ in M1B that requires $61 \%$ of patients treated in each department, would result in a decrease in the total number of treated patients.

\subsection{Severity of attack rate}

WHO estimates approximately $20 \%$ of the symptomatic cases to be severe ones (WHO 2015a). The Cuban Medical Brigade, which served $18 \%$ of the cholera cases in Haiti, asserts however that $4.8 \%$ of the total recovered cases were severely dehydrated (Lopez and Valido 2011). As there is no clear consensus for an accurate level of "severely ill patients", we analyze in this section the results obtained when the severity of the attack rate (i.e., proportion of severely ill patients) is in the range [5, 20\%]. Figure 4 shows that the medical staff availability levels in the base case are sufficient to treat $100 \%$ of the patients if the percentage of severely ill patients is $5 \%$ as argued by the Cuban Medical Brigade. As the proportion of severely ill patients increases, the ability to treat large numbers of patients lessens rapidly.

\subsection{Allocation of donations}

In this section, we propose a model that can be used by non-profit organizations to determine the best possible allocation of a received donation. Unlike in-kind material donations which are often unsolicited and pour in massive amounts (Özpolat et al. 2015), large institutional donors often earmark their monetary donations for a specific country, resource, or activity 


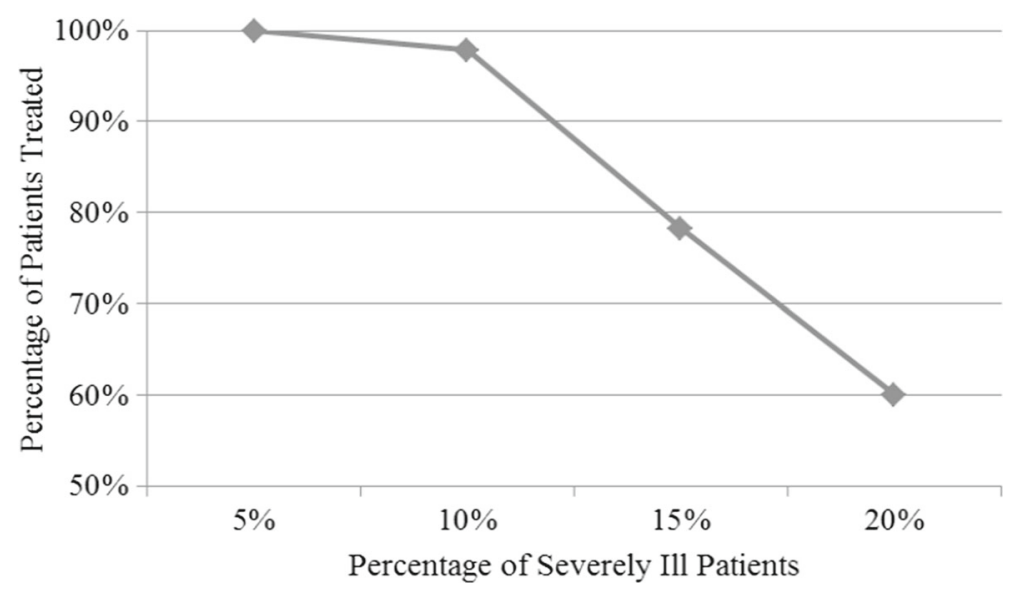

Fig. 4 Proportion of patients treated based on percentage of severely ill patients

(Bhattacharya et al. 2014). For example, the United States Agency for International Development (USAID) and the European Community Humanitarian Office (ECHO) earmark the funds they allocate for disaster operations (Besiou et al. 2014). However, as pointed out by Besiou et al. (2014), models for disaster relief operations hardly consider the aspect of such earmarked funding.

We consider in this section the case in which all in-country resources are fully utilized and model the effect of receiving earmarked donations on the opening and location of new facilities and on the funding for additional doctors and nurses. The model allows nonprofit organizations (e.g., International Committee of the Red Cross 2015) to identify and define a list of earmarked donation programs for particularly critical resources or projects. We specify the budget constraint in (21) where the superscript $h$ in each variable denotes newly hired staff, constructed facilities or new ambulances acquired through the donation budget:

$$
\begin{aligned}
& \sum_{j \in J}\left(7200 * z_{j 1}^{h}+5700 * z_{j 2}^{h}\right)+\sum_{j \in J} \sum_{l \in l}\left(5000 * x_{j 1}^{h}+1000 * x_{j 2}^{h}\right) \\
& \quad+\sum_{i \in I} \sum_{k \in K} 20000 * y_{i k}^{h} \leq B
\end{aligned}
$$

The constraints (3), (12), and (13) must be adjusted to account for new resources. The upper bounds on the number of staff and ambulances must also be increased due to the possibility of providing additional resources. The budget available to add new resources is $B$. The fixed cost to set up and operate a CTC was estimated by Schaetti et al. (2012) to be $\$ 51$ per patients and includes employee opportunity costs. Accordingly, we estimate the set up cost of a 100bed CTC and 20-bed CTU to be $\$ 5000$ and $\$ 1000$ respectively. MSF (2015) estimates the 3 -month salary of a nurse and a physician to $\$ 5700$ and $\$ 7200$. The purchase of an ambulance is set to $\$ 20,000$.

We consider donation budgets that vary between $\$ 250,000$ and $\$ 700,000$ by increments of $\$ 50,000$. We solve model M1 in which we add constraint (21) to determine the best possible allocation of the budget with the objective to treat as many severely ill patients as possible. Figure 5 shows the variation in the number of severely ill patients treated under each budget amount. Figure 6 indicates the allocation of the budget to the various 


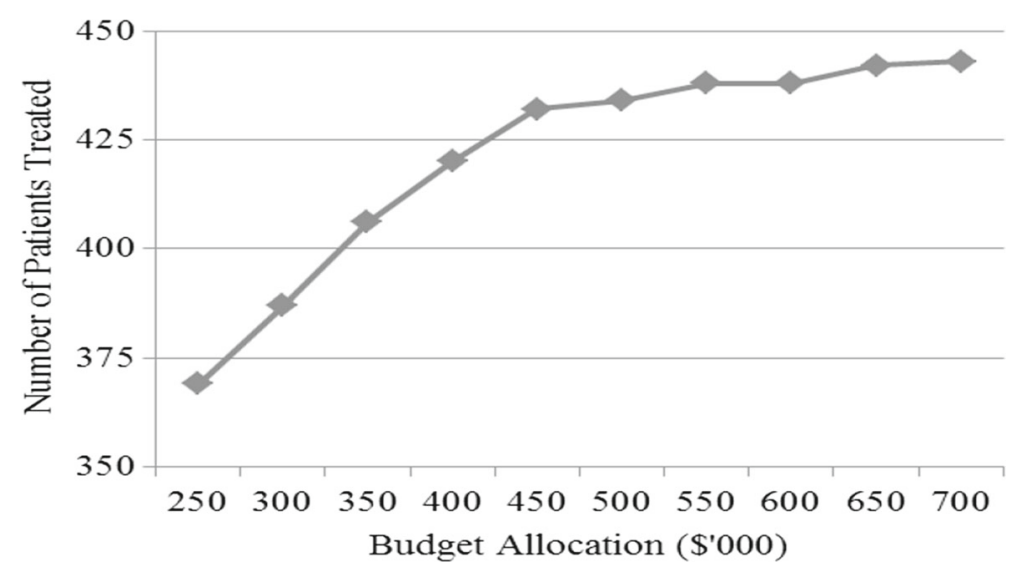

Fig. 5 Number of severely ill patients treated based on budget allocation

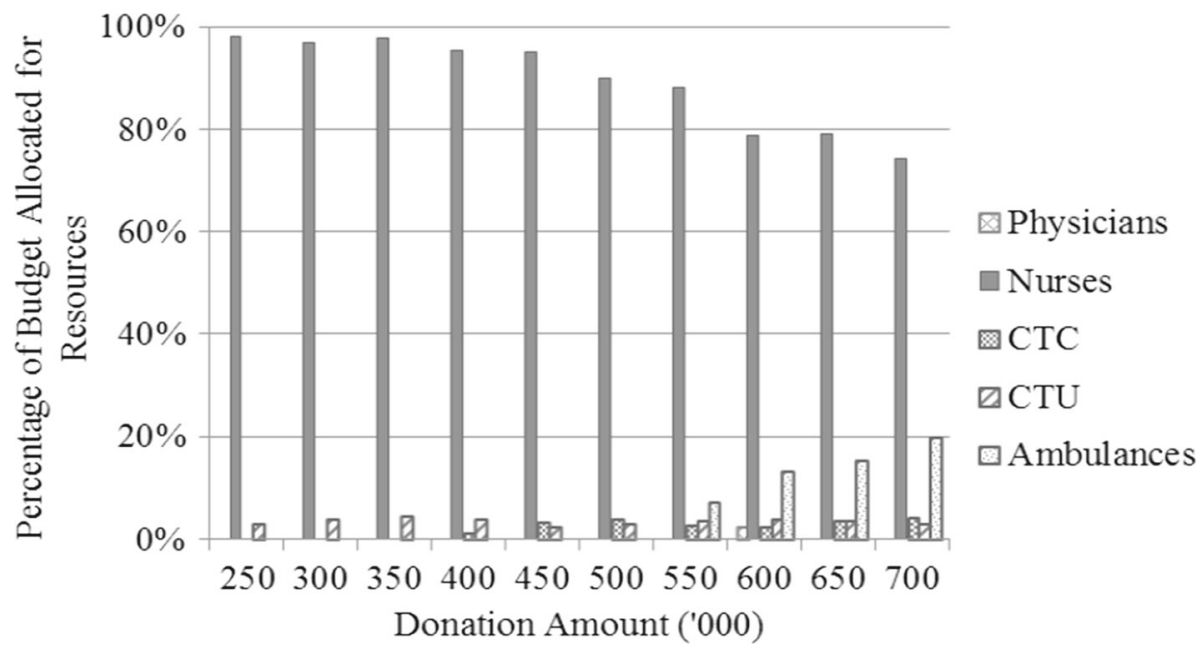

Fig. 6 Usage of donation

resources. As the donation amount increases, we observe diminishing marginal benefits in terms of the number of patients treated. Figure 6 shows that the largest proportion of the budget is systematically allocated to the hiring of nurses. Ambulance purchases only occur for budget levels exceeding $\$ 500,000$ that allow treating more than 438 patients. An additional budget of $\$ 700,000$ supplementing the resources specified in the base case scenario would permit the treatment of all 443 severely ill patients. This budget would be used to set up 6 additional CTCs, 11 more CTUs, purchase 7 ambulances, and hire 91 additional nurses.

\subsection{Comparisons with other cholera intervention strategies}

In this section, we compare the effect of our model with intervention strategies involving antibiotics, vaccination, and provision for clean water. Andrews and Basu (2011) analyzed the change in reported cholera case numbers with any single and combination of those strategies. 


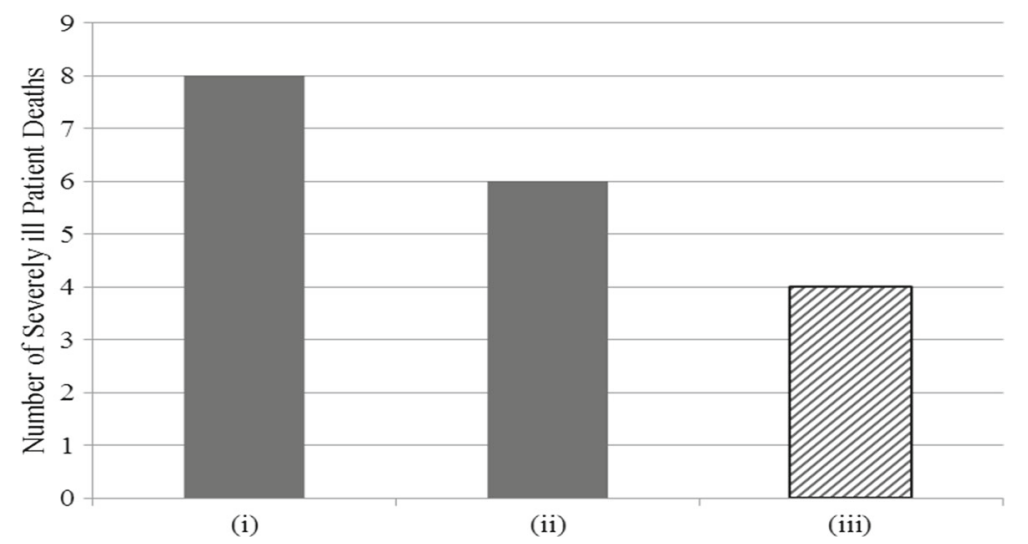

Fig. 7 Number of severely ill patient deaths in each scenario

We compare and compute the number of deaths for: Case (i) without any intervention; Case (ii) after the three interventions preconized by Andrews and Basu (2011); Case (iii) with Model M1 and availability of resources defined for the base case scenario. When comparing the effectiveness of the interventions, we must keep in mind that the morbidity and mortality results reported by Andrews and Basu (2011) are obtained via simulation, and are not based on actual patient number data reported for Haiti.

Andrews and Basu (2011) report a daily average of 2833 patients across Haiti for the March 2011-November 2011 period and provide the breakdown of case count per department. Twenty percent of the patients are considered severely ill (WHO 2015a). The total number of deaths due to cholera in Cases (i) and (ii) are directly obtained from Table 1 in Andrews and Basu (2011), and are equal to 40 and 28 per day respectively.

To compute the number of deaths with our intervention approach based on Model M1, we use the number of cholera patients provided by Andrews and Basu (2011) and employ the WHO CHOICE model (WHO 2015a) to estimate cholera treatment probabilities for the entire population. The probability of receiving treatment in a limited-resource country is set to 0.75 (WHO 2015b; Ali et al. 2012), when patient transportation is not provided. While the severely ill patients transported to properly staffed CTCs and CTUs receive treatment on time in our model, it is unsure whether it will be the case for patients who reach a treatment center by their own means. Hence, they are subject to the case fatality rate (CFR) set to $1.4 \%$ (Andrews and Basu 2011). As displayed in Fig. 7, the daily average of severely ill patient deaths in our approach [Case (iii)] is 4 and decreases by one third (resp., by half) the number of deaths in Case (ii) with Andrews and Basu's intervention strategies (resp., in Case (i) without any intervention).

This result highlights the importance and benefit of providing transport and properly allocating medical resources to CTCs and CTUs. The post-outbreak implementation of the interventions in Case (ii) is likely to be complicated to implement over the short-term in a resource-limited country which lacks vaccine stockpiles and has no pre-identified supply chain network (Maskery et al. 2013). Maskery et al. (2013) also note that the current vaccination "manufacturing capacity is limited and insufficient to properly respond to large, nation-wide outbreaks." Furthermore, poor water and sanitation conditions in countries such as Haiti make interventions involving the provision of clean water effective if planned and executed over the long term (Reuters 2015). 
Table 5 Number of severely ill patient deaths, direct and indirect costs under each case

\begin{tabular}{llllr}
\hline Case & $\begin{array}{l}\text { Daily number } \\
\text { of deaths }\end{array}$ & $\begin{array}{l}\text { 3-Month number } \\
\text { of deaths }\end{array}$ & $\begin{array}{l}\text { Value of lost productivity } \\
\text { over 3 months }(\$ 000)\end{array}$ & Benefits $b(j)\left(\$^{\prime} 000\right)$ \\
\hline (i) & 8 & 720 & 6462.92 & 0.00 \\
(ii) & 6 & 540 & 4847.19 & 1615.73 \\
(iii) & 4 & 360 & 3231.46 & 3231.46 \\
\hline
\end{tabular}

\subsection{Cost-benefit analysis}

In this section, we develop a cost-benefit analysis based on the cholera cost calculation guidelines set by the University of Pittsburgh Medical Center (UPMC) center for health security (UPMC 2014). We use a present discounted value approach that evaluates the epidemic's indirect costs expressed in terms of value of lost productivity due to lost human lives consecutive to an epidemic. The value of lost productivity $L$ is calculated as follows:

$$
L=n * \text { annual GDP per capita } /(1+v)^{n}
$$

where $n$ is the life years lost, $v$ is the discount rate set to $3 \%$ (Ali et al. 2012), and the 2011 annual GDP per capita in Haiti is $\$ 749$ (World Bank 2015). UPMC (2014) computes the life years lost as:

$$
n=0.8 *(g-15)+0.2 *(g-30)
$$

where $g$ is the country life expectancy and is estimated to be 62 years in Haiti (WHO 2015c). Since cholera-caused death rates are disproportionate among adults and children (MSPP 2011; UPMC 2014), a higher (resp., lower) weight 0.8 (resp., 0.2) is given to the life years lost due to the death of a young child (resp., adult) (UPMC 2014).

We measure the benefits $b(j)$ of an intervention strategy $j$ as the difference between the value of lost productivity $L(0)$ without any intervention (Case i) and the value of lost productivity $L(j)$ with intervention $j$ :

$$
b(j)=L(0)-L(j)
$$

In this study, the life years lost $n$ is 44 years, while the value of lost productivity $L$ is equal to $\$ 8976$ per dead person. Columns 2 and 3 in Table 5 report the average daily and 3-month numbers of deaths for each case. Column 4 provides the total value of lost productivity over 3 months. Column 5 indicates the value of lost productivity benefits. The benefits provided by the intervention defined by our model [Case (iii)] amounts to $\$ 3,231,460$ over 3 months and are almost twice as large as the benefits generated with the interventions of Case (ii). This is another testimony of the huge short-term impact that the proper allocation of medical and transportation resources has to control an epidemic outbreak in a resource-limited country.

We now apply the above framework to compute the benefits of a donation. In Sect. 5.5 (see Fig. 5), we computed the number of lives saved with a certain donation amount when this donation complements the intervention strategy defined by Model M1. We can then obtain (Table 6) the benefits expressed in terms of saved value of lost productivity for each donation amount. A $\$ 300,000$-donation permits to save 271 lives over 3 months and generates a benefit of $\$ 2,434,000$, while a $\$ 700,000$-donation provides saved lost productivity benefits of $\$ 3,215,000$. 
Table 6 Benefit of donation amounts

\begin{tabular}{lll}
\hline Donation value (\$) & Lives saved (3 months) & $\begin{array}{l}\text { Benefit from saved productivity } \\
\text { to GDP (\$ '00,000) }\end{array}$ \\
\hline 300,000 & 271 & 24.34 \\
500,000 & 292 & 26.24 \\
700,000 & 358 & 32.15 \\
\hline
\end{tabular}

\section{Efficiency of algorithmic method}

In this section, we assess the contribution of the proposed algorithmic method. In addition to the problem instances analyzed in the previous section, we have generated larger and more complex instances to check if the model can be efficiently and exactly solved through the incorporation of the hierarchical symmetry-breaking constraints and valid inequalities derived in Sect. 3.

The tests are conducted on 36 problem instances that differ in terms of the number $q$ of ambulances available, the number of physicians $\left(r_{1}\right)$ and nurses $\left(r_{2}\right)$, and the maximal traveling distance $d$ admissible between a triage location and a cholera treatment center. We consider three possible levels for $q$ and $d: q$ can take value 25,35 , and 45 , while $d$ can take value $0.5,1$, and $1.5 \mathrm{~h}$. Four values $(45,81),(30,54),(80,144)$, and $(150,270)$ are considered for the tuple $\left(r_{1}, r_{2}\right)$. We construct the full factorial design of experiments, which provides us with 36 problem instances. The AMPL modeling language is used to formulate the mathematical programming problems. The mixed-integer programming formulations are solved with the Cplex 12.6.1. Each problem instance is solved on a 64-bit Dell Precision T5400 Workstation with Quad Core Xeon Processor X5460 3.16GHz CPU. We allow $1 \mathrm{~h}$ of CPU time for each problem instance and with model.

We compare four modeling and algorithmic approaches. We first solve the model M1 with the Cplex solver. Next, we use branch-and-bound (B\&B) algorithms to solve: (1) model M1-H obtained by adding the hierarchical symmetry-breaking constraints (15) in M1; (2) model M1-V obtained by adding the valid inequalities (16)-(19) in M1; and (3) MI-HV in which the valid inequalities (16)-(19) and the hierarchical constraints (15) are incorporated.

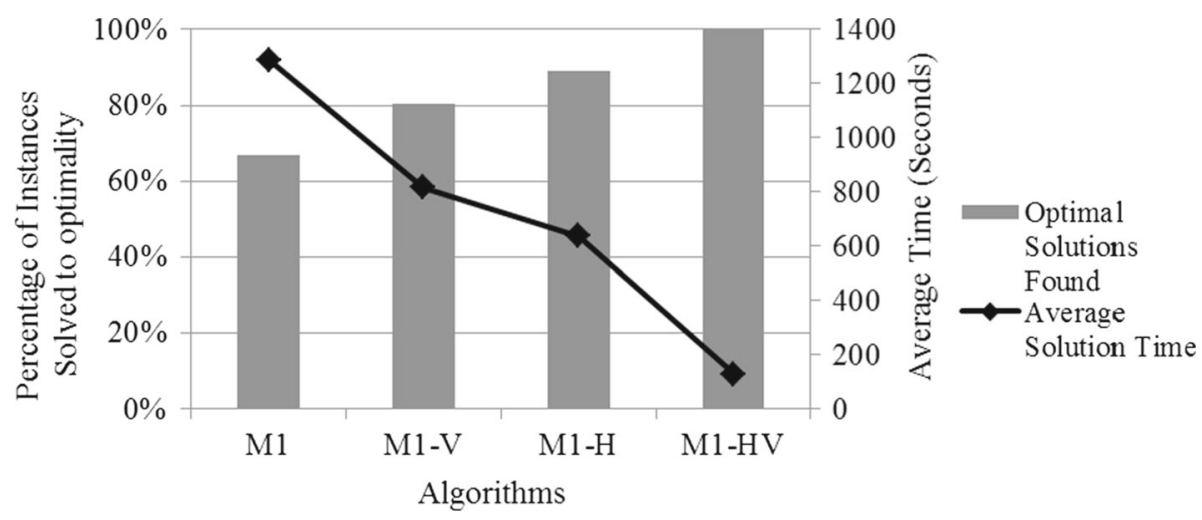

Fig. 8 Computational results 
Figure 8 displays the average solution time (in seconds and over the 36 instances) to prove the optimality of the solution and the number of problem instances solved to optimality. If optimality is not proven within $1 \mathrm{~h}$, we consider the time to be $3600 \mathrm{~s}$.

Figure 8 does not leave any doubt about the computational benefits of the proposed procedure. Model M1-HV is clearly the best since our B\&B algorithm solves and proves optimality for all instances within $20 \mathrm{~min}$. The longest solution time is $1084 \mathrm{~s}$, while the average solution time is $129 \mathrm{~s}$. The algorithmic method is about 10 (resp., 8 and 5) times faster than the Cplex solver (resp., the B\&B algorithm using valid inequalities only and using hierarchical constraints only) which takes on average 1287 (resp., 817 and 636)s. This highlights the benefits and scalability of the proposed approach. The added value of the M1-HV-based $\mathrm{B} \& \mathrm{~B}$ algorithm is particularly marked for the large and complex instances. Note that all the proposed algorithms (M1-HV, M1-H, and M1-V) significantly outperform the Cplex solver.

\section{Conclusions}

In developing countries, the consequences of epidemics are exacerbated due to poor water and sanitation conditions, and lack of access to health care services. This study proposes a resource allocation model for an epidemic outbreak occurring in the aftermath of a natural disaster in a resource-limited country. The model determines the number, size, and location of treatment facilities, deploys medical staff, locates ambulances to triage points, and organizes the transportation of geographically-dispersed severely ill patients to treatment facilities. The model is based on the 2010 cholera outbreak in Haiti, but is versatile enough to be applied and evaluate the response to other epidemic outbreaks.

Non-profit organizations play a central role in the response to an epidemic. The proposed model enables them to assess health care triage capabilities, transportation needs, and requirements for medical personnel. The model is widely applicable, as it complies with standards specified by regulatory bodies (i.e., WHO, CDC and MSPP) and accounts for the scarcity of the transportation and medical resources in developing countries, in particular in the aftermath of a natural disaster. This is important, since, as noted by Dasaklis et al. (2012), very few models for the control of epidemics in the aftermath of natural disasters are available, and those are likely to become more frequent (Kouadio et al. 2012). The continuity of operations aspect is also taken into account by opening CTCs and CTUs in the close vicinity of hospitals. While most epidemic models do not consider patient flow, we use a pull modeling approach that plans the transport of severely ill patients from triage locations to treatment facilities. We make sure that transportation occurs within reasonable delay through the incorporation of coverage constraints. The quick transportation of patients is motivated by the short incubation periods of some epidemics and the limited access to medical facilities in developing economies.

Additionally, we develop a framework that not-for-profit organizations can use to optimally allocate a donation and to determine a list of priorities for earmarked donations. We also propose a present discounted value model approach that permits to assess the benefitsexpressed in terms of saved value of lost productivity - of an intervention strategy financed by a donation. This will support not-for-profit organizations to design and prioritize (i.e., purchase of ambulances, medicine, tents, recruiting of medical personnel) their aid and donation policies.

The model takes the form of a complex integer linear programming problem with many symmetries. The proposed algorithmic procedure relies on hierarchical symmetry-breaking 
constraints and families of valid inequalities, and can be used for a variety of resource deployment problems with symmetries. The method permits to solve all instances to optimality quickly, and decreases the computational time by one order of magnitude as compared to the integer solver Cplex.

The analysis of Haiti's cholera outbreak on the basis of the data available to us reveals that the availability of nurses and physicians is the most critical resource that impacts most the number of patients that can be treated. This can come as somewhat of a surprise as some of the literature about the Haiti epidemics attributes a large number of deaths to the lack of transportation means (Farmer et al. 2011; Ivers et al. 2010). The number of treatment facilities is a decreasing function of the coverage requirement. The introduction of a service constraint permits to enforce a fair and balanced response across the country. However, due to the tightness of the available resources, the balanced response strategy reduces the overall welfare (i.e., total number of treated patients) if the fairness threshold is set too high. We also show how the response strategy can be modulated depending on the severity of the attack rate of the disease and under which conditions and to which extent the current resources must be supplemented. The comparison of the benefits provided by our approach with other intervention strategies highlights the importance of an appropriate allocation of medical and transportation resources on the treatment of severely ill patients. It is also important to remember that the proposed recommendations are based on the available data, which, in the context of a major disaster in a least country, are incomplete and could lead to some bias (e.g., underreporting of cholera cases in rural areas).

The proposed model responds to the call of Thompson et al. (2006) to develop robust models and decision support systems for disaster management, and addresses the need to jointly consider the allocation of limited medical (staff and medicine) and transportation resources, the transportation of patients to treatment centers, and the utilization of donations. We acknowledge that the applicability of a model similar to the one proposed in this study might be challenging, since it implicitly assumes the presence of a central planner in an environment where multiple organizations operate with almost no structure in place (Pedreza Martinez et al. 2011).

\section{References}

Aaby, K., Herrmann, J. W., Jordan, C. S., Treadwell, M., \& Wood, K. (2006). Montgomery County's public health service uses operations research to plan emergency mass dispensing and vaccination clinics. Interfaces, 36(6), 569-579.

AHRQ. (2004). Agency for Healthcare Research and Quality. Bioterrorism and Other Public Health Emergencies: Tools and Models for Planning and Preparedness: Community-Based Mass Prophylaxis, A Planning Guide for Public Health Preparedness.

Ali, M., Lopez, A. L., You, Y. A., Kim, Y. E., Sah, B., Maskery, B., et al. (2012). The global burden of cholera. World Health Organization Bulletin, 90, 209-218.

Altay, N., \& Green, W. G. (2006). OR/MS research in disaster operations management. European Journal of Operational Research, 175(1), 475-493.

Altay, N., \& Labonte, M. (2014). Challenges in humanitarian information management and exchange: Evidence from Haiti. Disasters, 38(s1), S50-S72.

Andrews, J. R., \& Basu, S. (2011). Transmission dynamics and control of cholera in Haiti: An epidemic model. The Lancet, 377(9773), 1248-1255.

Apte, A., Heidtke, C., \& Salmerón, J. (2015). Casualty collection points optimization: A study for the District of Columbia. Interfaces, 45(2), 149-165.

Besiou, M., Pedraza-Martinez, A. J., \& Van Wassenhove, L. N. (2014). Vehicle supply chains in humanitarian operations: Decentralization, operational mix, and earmarked funding. Production and Operations Management, 23(11), 1950-1965. 
Bhattacharya, S., Hasija, S., \& Van Wassenhove, L. N. (2014). Designing efficient infrastructural investment and asset transfer mechanisms in humanitarian supply chains. Production and Operations Management, 23(9), 1511-1521.

CDC. (2015). 2014 Ebola Outbreak in West Africa-Case Counts. http://www.cdc.gov/vhf/ebola/outbreaks/ 2014-west-africa/case-counts.html. Accessed March 10, 2015.

Dasaklis, T. K., Pappis, C. P., \& Rachaniotis, N. P. (2012). Epidemics control and logistics operations: A review. International Journal of Production Economics, 139(2), 393-410.

Drezner, T. (2004). Location of casualty collection points. Environment and Planning C: Government and Policy, 22(6), 899-912.

Dubey, R., \& Gunasekaran, A. (2016). The sustainable humanitarian supply chain design: Agility, adaptability and alignment. International Journal of Logistics Research and Applications, 19(1), 62-82.

Farmer, P., Almazor, C. P., Bahnsen, E. T., Barry, D., Bazile, J., Bloom, B. R., et al. (2011). Meeting cholera's challenge to Haiti and the world: A joint statement on cholera prevention and care. PLoS Neglected Tropical Diseases, 5, 1-13.

Fisher, M. L. S., Wood, T., \& Plyler, L. (2014). Responding to the cholera epidemic in Haiti. American Journal of Nursing, 114(5), 38-46.

Geohive. (2015). Haiti-General information. http://www.geohive.com/cntry/haiti.aspx.

Ghoniem, A., \& Sherali, H. D. (2011). Defeating symmetry in combinatorial optimization via objective perturbations and hierarchical constraints. IIE Transactions, 43, 575-588.

Google. (2015). Google Maps Image APIs. Google developers. https://developers.google.com/maps/ documentation/staticmaps/. Accessed February 25, 2015.

Harewood, S. I. (2002). Emergency ambulance deployment in Barbados: A multi-objective approach. Journal of the Operational Research Society, 53(2), 185-192.

International Committee of the Red Cross (ICRC). (2015). ICRC Resource Center. https://www.icrc.org/en/ resource-centre. Accessed April 23, 2015.

Ivers, L. C., Farmer, P., Almazor, C. P., \& Léandre, F. (2010). Five complementary interventions to slow cholera: Haiti. The Lancet, 376(9758), 2048-2051.

Kouadio, I. K., Aljunid, S., Kamigaki, T., Hammad, K., \& Oshitani, H. (2012). Infectious diseases following natural disasters: Prevention and control measures. Expert Review of Anti-infective Therapy, 10, 95-104.

Koyuncu, M., \& Erol, R. (2010). Optimal resource allocation model to mitigate the impact of pandemic influenza: A case study for Turkey. Journal of Medical Systems, 34(1), 61-70.

Kraiselburd, S., \& Yadav, P. (2013). Supply chains and global health: An imperative for bringing operations management scholarship into action. Production and Operations Management, 22(2), 377-381.

Lee, E. K., Smalley, H. K., Zhang, Y., \& Pietz, F. (2009). Facility location and multi-modality mass dispensing strategies and emergency response for biodefence and infectious disease outbreaks. International Journal of Risk Assessment and Management, 12(2), 311-351.

Lemonick, D. M. (2011). Epidemics after natural disasters. American Journal of Clinical Medicine, 8(3), $144-152$.

Lopez, L. S., \& Valido, J. A. S. (2011). Cholera epidemic in Haiti: Experience of the Cuban Medical Brigade. Revista Cubana de Salud Pública Internacional,2(1). http://www.medigraphic.com/pdfs/ revcubsalpubint/spi-2011/spi111h.pdf. Accessed October 20, 2014.

Margot, F. (2010). Symmetry in integer linear programming. In M. Junger, T. M. Liebling, D. Naddef, G. Nemhauser, W. R. Pulleyblank, G. Reinelt, G. Rinaldi, \& L. A. Wolsey (Eds.), 50 Years of integer programming (pp. 647-686). Berlin: Springer.

Maskery, B., DeRoeck, D., Levin, A., Kim, Y. E., Wierzba, T. F., \& Clemens, J. D. (2013). Strategy, demand, management, and costs of an international cholera vaccine stockpile. Journal of Infectious Diseases, 208(1), S15-S22.

McCoy, J. H., \& Johnson, M. E. (2014). Clinic capacity management: Planning treatment programs that incorporate adherence. Production and Operations Management, 23(1), 1-18.

McCoy, J. H., \& Lee, H. L. (2014). Using fairness models to improve equity in health delivery fleet management. Production and Operations Management, 23(6), 965-977.

McLachlin, R., Larson, P., \& Khan, S. (2009). Not-for-profit supply chains in interrupted environments. Management Research News, 32(11), 1050-1064.

MSF. (2011). Haiti one year after. Medecins Sans Frontieres. http://www.doctorswithoutborders.org/sites/usa/ files/Haiti-one-year-report.pdf. Accessed March 4, 2015.

MSF. (2015). Benefits and Opportunities. Médecins Sans Frontières. http://www.doctorswithoutborders.org/ workus/work-field/benefits-opportunities. Accessed February 18, 2015.

MSPP. (2011). Documentation. Ministry of Health and Population, Haiti. http://www.mspp.gouv.ht/site/index. php?option=com_content $\&$ view=article $\&$ id=57\&Itemid=1. 
MSPP and CDC. (2011). Haiti Cholera Training Manual: A Full Course for Health Care Providers.http:// www.cdc.gov/haiticholera/pdf/haiticholera_trainingmanual_en.pdf. Accessed August 14, 2014.

Özpolat, K., Rilling, J., Altay, N., \& Chavez, E. (2015). Engaging donors in smart compassion: USAID CIDI's Greatest Good Donation Calculator. Journal of Humanitarian Logistics and Supply Chain Management, 5(1), 95-112.

PAHO. (2010). Haiti-Health facilities: Monitoring health service response capacity. http://ais.paho.org/phip/ viz/haiti_healthfacilities_v2.asp. Accessed September 2, 2014.

Pedreza Martinez, A. J., Stapleton, O., \& Van Wassenhove, L. N. (2011). Field vehicle fleet management in humanitarian operations: A case-based approach. Journal of Operations Management, 29(5), 404-421.

PRI. (2014). Emergency medical care is a rare but fragile sign of progress in Haiti. Public Radio International (PRI). http://www.pri.org/stories/2014-10-21/emergency-medical-care-rare-fragile-signprogress-haiti. Accessed February 18, 2015.

Rachaniotis, N. P., Dasaklis, T. K., \& Pappis, C. P. (2012). A deterministic resource scheduling model in epidemic control: A case study. European Journal of Operational Research, 216(1), 225-231.

Rahman, S., \& Smith, D. K. (2000). Use of location-allocation models in health service development planning in developing nations. European Journal of Operational Research, 123, 437-452.

Reuters. (2015). Cholera will plague Haiti until water, sanitation crisis solved: Experts. http://www.reuters. com/article/2015/03/26. Accessed April 6, 2015.

Roos, R. (2013). Cholera has struck more than 6\% of Haitians. Center for Infectious Disease Research and Policy (CIDRAP). http://www.cidrap.umn.edu/newsperspective/2013/01/cholera-has-struck-more6-haitians. Accessed March 10, 2015.

Schaetti, C., Mitchell, G., Said, M., Claire-Lise, C., Ahmed, M., Rita, et al. (2012). Costs of illness due to cholera, costs of immunization and cost-effectiveness of an oral cholera mass vaccination campaign in Zanzibar. PLoS neglected tropical diseases, 6(10), e1844:1-10.

Sherali, H. D., \& Smith, J. C. (2001). Improving discrete model representations via symmetry considerations. Management Science, 47, 1396-1407.

Sobieraj, J. A., Reyes, J., Dunemn, K. N., Carty, I. H., Pennathur, A., Gutierrez, R. S., et al. (2007). Modeling hospital response to mild and severe influenza pandemic scenarios under normal and expanded capacities. Military Medicine, 172(5), 486-490.

Thompson, S., Altay, N., Green, W. G, I. I. I., \& Lapetina, J. (2006). Improving disaster response efforts with decision support systems. International Journal of Emergency Management, 3(4), 250-263.

UN. 2010. Report of the United Nations in Haiti 2010: Situation, challenges and outlook. United Nations Organization (UN). http://www.un.org/en/peacekeeping/missions/minustah/documents/un_ report_haiti_2010_en.pdf. Accessed February 20, 2015.

UNDP. (2009). Human development report: Overcoming barriers: Human mobility and development. http:// hdr.undp.org/en/media/HDR_2009_EN_Complete.pdf.

UNICEF. (2013). At a Glance: Haiti. http://www.unicef.org/infobycountry/haiti_statistics.html.

UPMC. (2014). Infectious disease cost calculator: Cholera cost model methods. UPMC Center for Health Security. http://www.idcostcalc.org/contents/cholera/cost-model.html. Accessed April 5, 2015.

Van Wassenhove, L. N., \& Pedraza Martinez, A. J. (2012). Using OR to adapt supply chain management best practices to humanitarian logistics. International Transactions in Operational Research, 19(1-2), 307-322.

World Bank. (2015). World Data Bank. http://databank.worldbank.org/data/views/reports/tableview.aspx.

World Health Organization (WHO). (2004). Cholera outbreak: Assessing the outbreak response and improving preparedness. In Cholera outbreak: Assessing the outbreak response and improving preparedness. http:// www.who.int/cholera/publications/OutbreakAssessment/en/. Accessed April 5, 2015.

WHO. (2015a). Cholera fact sheet. World Health Organization. http://www.who.int/mediacentre/factsheets/ fs107/en/. Accessed December 22, 2014.

WHO. (2015b). Cost effectiveness and strategic planning (WHO-CHOICE). World Health Organization. http:// www.who.int/choice/en/. Accessed April 10, 2015.

WHO. (2015c). Haiti: Statistics. World Health Organization. http://www.who.int/countries/hti/en/.

World Bank. (2015). Physicians (per 1000 people). Data: The World Bank. http://data.worldbank.org/ indicator/SH.MED.PHYS.ZS?page=3. Accessed January 10, 2015. 